Manuscript submitted to editorial office

International Journal

WPS ENHANCES INTER-SEGMENTAL FOOT COORDINATION

\begin{tabular}{|r|l|}
\hline Journal: & International Journal of Sports Medicine \\
\hline Manuscript ID: & IJSM-02-2012-2667-ob.R2 \\
\hline Manuscript Type: & Orthopedics \& Biomechanics \\
\hline Key word: & $\begin{array}{l}\text { Rehabilitation, Wide-pulse electrical stimulation, Foot, Kinematic coupling, } \\
\text { Post-tetanic potentiation }\end{array}$ \\
\hline \multicolumn{2}{|r}{} \\
\hline
\end{tabular}

3

4

5

6

7

8

9

10

11

12

13

14

15

16

17

18 


\section{Wide-pulse electrical stimulation to an intrinsic foot muscle induces acute functional changes in}

\section{forefoot - rearfoot coupling behaviour during walking.}

Darren C James ${ }^{12}$, Tom Chesters ${ }^{3}$, D Paul Sumners ${ }^{12}$, David P Cook ${ }^{2}$, David A Green ${ }^{3}$ and Katya N

Mileva $^{2}$

${ }^{1}$ Human Performance Centre, London South Bank University, UK.

${ }^{2}$ Faculty of Engineering, Science \& the Built Environment, London South Bank University, UK.

${ }^{3}$ Centre for Human \& Aerospace Physiological Sciences, King’s College London, UK.

Email: jamesd6@1sbu.ac.uk Web: www.lsbu.ac.uk/sports/hpc

8

\section{Abstract}

Interventions for strengthening intrinsic foot muscles may be beneficial for rehabilitation from overuse injuries. In this study the acute effects of high-frequency, low-intensity wide-pulse electrical stimulation (WPS) over an intrinsic muscle on subsequent foot function during walking was assessed in healthy participants. WPS was delivered to the $m$. abductor hallucis ( $m . \mathrm{AH}$ ) of the non-dominant foot during relaxed standing. Three-dimensional forefoot $(\mathrm{FF})$ - rearfoot $(\mathrm{RF})$ coordination was quantified with a vector coding technique within separate periods of the stance phase to study WPS functional effects on foot motion. Four types of coordinative strategies between the FF and RF were interpreted and compared PRE-to-POST-WPS for both the experimental and control feet. Bilateral electromyography (EMG) from m.AH was analysed during the intervention period for evidence of acute neuromuscular adaptation.

The results showed that WPS significantly modulated FF-RF coordination during mid-stance, indicative of a more stable foot. Specifically, a statistically significant increase in FF eversion with concomitant RF inversion in the frontal plane and RF-dominated adduction in the transverse plane was observed. Subjectspecific increases in post-stimulus m.AH EMG activation were observed but this was not reflected in an overall group effect. It is concluded that the structural integrity of the foot during walking is enhanced following an acute session of WPS and that this mechanical effect is most likely due to stimulation induced post-tetanic potentiation of synaptic transmission. 


\section{Introduction}

There is increasing evidence demonstrating the importance of the intrinsic foot muscles to longitudinal arch stability during locomotion $[2,7,13,15,18,22]$. Recently, the structural integrity of the arch has been suggested to be enhanced by the higher forces exerted by the intrinsic muscles of the foot during late stance [2]. The contribution of these muscles has been speculated to be greater in the pronated foot [18]. Insufficient pronation control is linked to several common overuse injuries, including plantar fasciitis, achilles tendonitis, hallux valgus and tibialis posterior tendonitis [15]. Orthotic intervention is frequently prescribed to assist in motion control and may also be extended to asymptomatic individuals. However, a recent review of the literature concluded that clinicians adopt a rudimentary approach in the prescription of orthoses due in part to substantial variability and a lack of homogeneity in the evidence base [19].

Moreover, the long term beneficial effects of orthoses have been questioned [16] although this may relate to inappropriate prescription [16].

Strengthening of the intrinsic foot musculature may be an efficient solution in the treatment/prevention of common foot-related disorders [7, 13, 15, 22]. Studies have demonstrated arch depression when $m$. abductor hallucis (m.AH) is paralysed following tibial nerve block [7] or fatigued following exercise [13]. Others have also observed the role of $m . \mathrm{AH}$ in elevating the arch with restriction of hallux range of motion [22]. Moreover, an acute session of neuromuscular electrical stimulation (NMES) to this muscle can elicit prolonged synaptic facilitation resulting in lasting postural adjustments [8].

NMES has been shown to increase neural activation and strengthen human skeletal muscle [9]. It has been endorsed as a compliment to voluntary exercise and further posited as a rehabilitative tool for pathology that compromise normal neuromuscular function [4]. Only recently have the cellular and molecular mechanisms responsible for the observed improvement in muscle function been elucidated. In both athletic and sedentary individuals, type I and type IIa fibre hypertrophy was found following an eight week NMES training intervention along with a shift in myosin heavy chain isoform distribution indicative of a fast-toslow phenotype transition. Up- and down regulation of myofibrillar, energy production and anti-oxidant defence proteins were also consistent with the reported change in muscle phenotype [9]. 
The effect of NMES may be enhanced by utilisation of high-frequency, low-intensity, wide-pulse stimulation (WPS). WPS has been shown to induce sustained depolarisation of spinal motoneurons (plateau potentials) caused by persistent inward currents (PICs) and is reported to recruit motor units according to the 'size-principle' unlike conventional NMES [4]. Furthermore, WPS of lower leg muscle consistently induces a two-fold increase in force output when $100 \mathrm{~Hz}$ stimulation precedes $20 \mathrm{~Hz}$ stimulation. Such force increments are proposed to be evidence of an enhanced contribution from central neural mechanisms [4]. WPS has previously been performed in recumbent subjects only; whether the reported induced neural plasticity produces a substantial functional effect during a dynamic activity such as walking, when spinal reflexes are both task and phase dependent [23] remains unknown.

In order to understand this adaptation from a biomechanical perspective, it would be intuitive to implement a kinematic approach that classifies forefoot-rearfoot coordination patterns since $m$. $\mathrm{AH}$ originates from the calcaneus and inserts at the base of the first phalanx of hallux. Dynamic systems analysis has been an emerging line of investigation for over a decade and offers an insight into the subtleties of movement coordination and stability that traditional time domain kinematic analysis cannot. Continuous relative phase (CRP) plots are one such measure and have been shown to be sufficiently robust to detect differences in lower extremity coordinative patterns between healthy subjects and individuals suffering from patellofemoral pain syndrome [10]. A surrogate of CRP is the vector coding technique, which allows the interpretation of kinematic coupling between adjacent segments and can be summarised into four distinct coupling patterns: anti-phase, in-phase, proximal phase and distal phase motion [3]. This method provides a $360^{\circ}$ representation of continuous coupling between adjacent segments, whereby a coupling angle is able to distinguish between phase relationships (anti-/in-phase) or distal/proximal segment dominance. Indeed, the complexity of inter-segmental foot motion has only been realised following the emergence of this technique when applied to forefoot-rearfoot coupling relationships during walking [3].

Therefore, the aim of the present study was to investigate the effect of an acute session of WPS applied to $m . \mathrm{AH}$ on forefoot-rearfoot coupling motion during walking. It was hypothesised that enhanced activation of $m$.AH would induce alterations in inter-segmental foot motion during the middle to late phases of 
stance, when the activation of this muscle is most pronounced, resulting in an increased stability of the foot.

\section{Methods}

Ten healthy subjects (mean \pm SD: 5 male: $32.2 \pm 5.3 y r s, 1.79 \pm 0.07 \mathrm{~m}, 83.7 \pm 19.7 \mathrm{~kg} ; 5$ female; $28.0 \pm$ $6.3 y r s, 1.65 \pm 0.02 \mathrm{~m}, 62.6 \pm 4.2 \mathrm{~kg}$ ) free from any lower extremity injury or pathology gave their written informed consent to participate in the study which had received local ethical approval and adhered to the recognised standards of the IJSM [11].

Subjects attended a familiarisation session on a separate day prior to experimental data collection. $m$.AH motor point and threshold were identified in the non-dominant foot (experimental foot) for optimal response and stimulation intensity, respectively. A 7x5cm matrix was drawn over the muscle with respect to the navicular tuberosity (NT) in accordance with the literature [6] (Figure 1). The correct position for anode (Ag/AgCl, Cardicare) placement within the matrix was determined through the response from a triaxial accelerometer (Biometrics Ltd, UK) attached to the dorsal aspect of the hallux to a $500 \mu$ s squarewave pulse of $200 \mathrm{~V}$ with increasing current. In most cases the motor point was located $3 \mathrm{~cm}$ inferior and $1 \mathrm{~cm}$ posterior to the NT. A cathode was positioned over the medial aspect of the distal first metatarsal and motor threshold was determined by delivering a $100 \mathrm{~Hz}$ train of $5 \mathrm{x} 1$-ms square wave pulses [4]. Current was increased in $0.1 \mathrm{~mA}$ increments until a visible spike indicative of induced muscle contraction (motor threshold) was registered by the accelerometer. The stimulation intensity for all subsequent interventions was then set at $150 \%$ of motor threshold. Subject's individual walking speed was ascertained from five preliminary barefoot walking trials at selfselected speed. All subsequent main trials were required to fall within this speed range (mean $\pm 1 \mathrm{SD}$ ). of alternating WPS $(20 \mathrm{~Hz}-100 \mathrm{~Hz}$ [high-frequency]-20Hz) (Figure 2). Square wave (1ms[wide-pulse]) pulses $(40 \mathrm{~V})$ were delivered to $m$.AH of the non-dominant foot (experimental) by a constant-current stimulator (DS7A, Digitimer, UK) and driven by a custom written sequencer (Spike 2, v6.10, Cambridge 
UK) at the pre-determined current level (150\% motor threshold, low-intensity). Stimulation was delivered during standing with the dominant foot serving as the control (Figure 1). Two minutes seated rest was given between each stimulation train.

Bipolar surface EMG electrodes (1mm width, 10mm pole spacing; Delysis Inc., USA) were located over the distal aspect of $m . \mathrm{AH}$ on each foot following the pre-intervention walking trials. The raw signal from each muscle was pre-amplified (x1000), sampled at $2 \mathrm{kHz}$ and recorded throughout each WPS session once the subject was comfortably standing until 30 seconds after the stimulus had ceased. The EMG sensors were then removed following the ten sessions of WPS so that the post-intervention kinematic measures could be acquired.

Thirteen retro-reflective markers (12mm diameter) using a six degree-of-freedom marker set were positioned on each lower limb and defined the shank, rearfoot, mid-foot, forefoot and hallux segments in accordance with an accepted multi-segment foot/shank model [17] (Figure 1). A further seven markers on each limb were placed on anatomical landmarks during static calibration in order to define the segment coordinate system [17]. Three-dimensional kinematic data were captured at $500 \mathrm{~Hz}$ using an eight-camera motion analysis system (Qualisys AB, Sweden) synchronised with data from two force platforms (Kistler, UK) imbedded into a walkway for the identification of heel-strike and toe-off. A total of 100 strides (10 subjects-5 trials) in each condition (PRE vs. POST) were extracted for further analysis.

Kinematic data were processed in Visual 3D (C-Motion Inc, USA). FF-RF segment angles were calculated relative to a fixed laboratory coordinate system using a Cardan XYZ sequence of rotations. Segmental angle-angle plots were derived in the sagittal, frontal and transverse planes of motion and time normalised to $100 \%$ stance phase. Coordination was inferred from a coupling angle $(\gamma)$ subtended from a vector adjoining two successive time points relative to the right horizontal axis, where $0 \leq \gamma \leq 360$ [3]. The coupling angle represents four unique coordination strategies identified as 1) anti-phase motion, 2) inphase motion, 3) proximal (RF) phase motion, and 4) distal (FF) phase motion. These are located on the $45^{\circ}$ diagonals, horizontal and vertical axes of the angle-angle plots respectively [3] (Figure 3). The summation of the frequency of observations of $\gamma$ for each phase/plane/time were plotted as histograms and sub-divided into three equal periods of stance: early (1-33\%), mid (34-66\%) and late (67-100\%). 
Medial longitudinal arch angle was defined for each foot in the sagittal plane as the projection of the lines extending from the calcaneus to the sustentaculum tali to the first metatarsal head [17]. Arch angle was measured at heel-strike, peak active load (loading response), minimum load (mid-stance), peak propulsive load (terminal stance) and toe-off instances of the vertical ground reaction force.

EMG of $m$.AH from both feet was analysed using a custom-written script developed in Spike 2 version 6.10 software (Cambridge Electronic Design Ltd., UK) for two seconds prior to and immediately following each stimulation train (Figure 2). Each signal was high-pass filtered at $20 \mathrm{~Hz}$ and the magnitude of muscle activation was assessed by calculating the root-mean square (RMS) of the filtered signal. The average difference between the ten PRE- and POST-WPS measures was calculated for each subject and expressed as percentage change $(\Delta \%)$ for statistical comparison to account for inter-subject variation in EMG amplitude.

Preliminary test-retest experiments based on the same protocol but without WPS intervention were performed on a gender and age-matched control group $(n=4)$ to assess the $95 \%$ confidence limits that any kinematic effect resulting from the WPS treatment would have to exceed for that effect to be considered meaningful [1]. These limits (95\%CI) are presented in parentheses with any significant interaction effects reported in the main data.

The kinematic data from the main experiment were confirmed as being normally distributed (Kolmogorov-Smirnov 1-sample t-test; SPSS v.14.0; SPSS Inc., USA). Hence, a two-way repeated measures ANOVA was used to identify interaction effects and effect sizes $\left(\eta^{2}\right)$ of two investigated factors (foot [experimental vs. control] $\mathrm{x}$ time [PRE- vs.POST-WPS]).. A paired sample t-test was applied to the EMG percentage changes ( $\Delta \%$, PRE- vs.POST-WPS) to identify differences between feet. Statistically significant differences were accepted when $P<0.05$.

\section{Results}

FF-RF kinematic coupling was found to be significantly modulated during mid-stance as a result of WPS. Specifically, a significant frontal plane anti-phase motion (interaction effect: $\mathrm{F}=9.30 ; P=0.014 ; \eta^{2}: 0.51$ ) was observed. FF eversion-RF inversion increased in the experimental foot (mean \pm SD: $5.4 \pm 6.7$ a.u; 
$95 \%$ CI: $1.0 \pm 4.2$ a.u $)$ with respect to pre-WPS but remained unchanged in the control foot $(0.4 \pm 5.8$ a.u; 95\% CI: $-1.8 \pm 13.3$ a.u) (Figure 4, left panel). In addition, a significant transverse plane proximal-phase motion (interaction effect: $\mathrm{F}=9.96 ; P=0.012 ; \eta^{2}: 0.53$ ) was found during this period. $\mathrm{RF}$ adduction in the experimental foot increased by $3.2 \pm 5.9$ a.u (95\% CI: $-2.0 \pm 6.6$ a.u) in contrast to an overall decrease of this motion in the control foot $(-1.7 \pm 7.9$ a.u; $95 \%$ CI: $-3.0 \pm 8.3$ a.u) (Figure 4, right panel). No other significant phase/plane/time differences were found.

There were no significant differences in arch angle at any of the time periods measured within the gait cycle; although there was a tendency toward a significant difference between the feet during loading response (peak load, interaction effect; $P=0.064$ ). Post stimulation, the arch angle of the experimental foot decreased by $-0.48 \pm 0.31^{\circ}$ whereas the angle of the control foot increased by $0.21 \pm 0.08^{\circ}$.

The amplitude change of the RMS EMG signal in the experimental foot (mean \pm SD: $54.0 \pm 127.3 \%$ ) was not significantly different compared to the control foot $(8.1 \pm 32.7 \%)$ following the WPS intervention $(P=0.285)$. Subject-specific responses were inconsistent although most participants demonstrated sustained m.AH activation in the experimental foot following stimulation cessation, albeit not throughout all ten WPS trials. Also, there was further evidence of contralateral compensatory activation in some subjects where enhanced $m$.AH activation of the control foot was observed in response to the WPS stimulation of the experimental foot. Two subjects failed to show any facilitation throughout the intervention whereas, two demonstrated enhanced $m$. AH activation by more than $220 \%$ in the experimental foot following stimulation, thereby contributing to the large but non-significant difference in RMS EMG amplitude change between the feet $(54.0 \%$ vs. $8.1 \%)$..

\section{Discussion}

To our knowledge, this is the first study to investigate the effects of high-frequency, low-intensity WPS on functional movement patterns during a daily task such as walking. There is considerable evidence in the literature that narrow-pulsed, high-frequency NMES applied over muscle during a voluntary contraction at a high-intensity elicits strength improvements mediated by cellular and molecular adaptations [9]. However, motor-unit recruitment is non-selective during this paradigm, as high-intensity electrical 
stimulation would directly depolarise all motor axons in addition to sensory muscle afferents under the stimulus area.. High-frequency, low-intensity WPS on the other hand is posited to recruit motor units in a more physiological manner (i.e. according to the size principle) [4]. The ability of WPS to maximize the sensory volley to spinal motoneurons through activation of large-diameter afferents is due to a longer strength-duration time constant and lower rheobase of sensory axons compared to motor axons [4]. As such WPS has been proposed as a rehabilitative tool in restoring muscle function.

In the present study WPS was applied to $m$. abductor hallucis ( $m . \mathrm{AH})$ in healthy subjects to investigate the efficacy of this modality as a possible complementary technique to the existing and apparently contentious treatment of common foot-related over-use injuries $[16,19]$. The rationale for specifying this specific muscle was based on 1) the increasing evidence highlighting the importance of this muscle amongst other intrinsic foot muscles in maintaining medial longitudinal arch stability [2, 7, 13, 15, 18, 22]; and 2) its superficial location for purposes of stimulation and acquisition of a reliable EMG signal. By delivering a $100 \mathrm{~Hz}$ train interspersed between $20 \mathrm{~Hz}$ trains [4], the intention was to induce a tetanic contraction of $m . \mathrm{AH}$, thereby increasing the overall contraction magnitude. For reasons of electrical interference produced by the WPS on the EMG signal, electrophysiological quantification of contraction could not be performed during the stimulation; however, based on visual inspection alone this was confirmed. In agreement with the literature, we observed a stimulation-evoked contraction in all subjects consistent with the action of $m . \mathrm{AH}$ (flexion/abduction of hallux), which in recumbent subjects has been shown to be facilitated further with the addition of high-frequency WPS [4]. Such behaviour is indicative of a post-tetanic potentiation (PTP) resulting from PICs activation due to hyperexcitability of motoneurons[4]. It was hypothesised in the present study that the effect of WPS on $m$.AH and subsequent hallux action would be retained during functional movement (walking) in accordance with the PTP theory, postulating that the potential at the presynaptic neuron of the afferent signal can be enhanced for minutes to hours following a period highfrequency stimulus activity [12].

The results of the present study imply that an acute session of WPS applied to $m$.AH induces functional alterations in subsequent foot kinematics during the mid-stance phase of gait. Specifically, the results suggest a more stable foot following the intervention with the observation of maintained FF eversion with 
concomitant RF inversion in the frontal plane and RF-dominated adduction in the transverse plane. These findings indicate an increased torsion of the plantar fascia [3] that is conducive for the transfer of force during push-off as a result of an enhanced mid-tarsal joint function [18]. Indeed, the importance of the adaptive nature of mid-foot kinematics during propulsion has been demonstrated as a compensatory adjustment in gait in response to delayed onset of muscle soreness [20]. Furthermore, the efficiency of propulsion is believed to depend on the magnitude of force exerted by the intrinsic foot muscles rather than plantar aponeurosis tension [2].

We can report with $95 \%$ confidence that the effects on frontal plane kinematics are due to the WPS intervention. However, whilst a significant interaction effect was noted for transverse plane RF-dominated motion; it cannot be confirmed at present whether this is functionally meaningful as it fell within the confidence interval calculated from the preliminary experiments. Moreover, a decrease in this motion was not expected in the control foot. This may have been attributed to a contralateral compensation during the WPS intervention whereby selected subjects shifted their centre of mass over the stimulated foot to counter-act an illusory perception orientated ipsilateral to the stimulated foot [21]. Indeed, such phenomena have been demonstrated during mechanical stimulation of the plantar surface of the foot during standing [21]. Whether the consequences of this postural adjustment during standing can be translated into functional movement pattern and provide a rational explanation for the aforementioned finding remains to be determined. However, plantar pressure analysis (unpublished data) shows increased post-stimulation contact area in the experimental foot indicative of postural adjustments due to the electrical stimulation of $m . \mathrm{AH}$

Vector coding of kinematic waveforms has received little attention in the literature. Its intricate nature may discourage some researchers from adopting this approach above traditionally-favoured time-domain methods. However, this method is well-suited for investigating inter-segmental foot coordinative patterns since its accuracy in identifying specific phase relationships (anti-/in-phase) or segmental dominated motion is able to provide a more in-depth understanding of intrinsic foot biomechanics [3]. The present FFRF data is in accordance with data previously reported [3]. Specifically, these authors showed no particular dominant FF-RF frontal plane motion during the mid-stance period of the gait cycle but forefoot motion 
was found to be greatest. The frequency of observations for all frontal plane FF-RF coordinative motions during mid-stance reported in the present study are in good agreement with this.. Furthermore, our transverse plane data concurs with a trend to overall FF-RF coordination during mid-stance albeit less inphase motion in favour of increased distal phase motion.

Further evidence in support of our findings may be gleaned from Gaillet et al. [8]. In this study the authors found that a 20 minute session consisting of four seconds of electrical stimulation, with $400 \mu$ s pulses at $80 \mathrm{~Hz}$ applied to $m$.AH during standing and interspersed with $6 \mathrm{~s}$ rest induced specific changes in baropodogram indices with immediate learning effects, some of which persisted following a two-month retention test [8]. The reported effects were of similar size to those found in the present study. PTP was proposed as a neural mechanism responsible for the retained postural effects. In the present study longer pulse duration (1 ms) was adopted since sensory axons are more effectively depolarised by wider pulsewidths [4]. In studies from this research group, post-stimulation plateau potential phenomena as a result of PICs activation have consistently been reported in recumbent subjects. However, in the present study only two subjects demonstrated measureable post-stimulation enhanced muscle activity during relaxed standing. Variability of PICs between subjects is well-documented and highly dependent on monoamine drive [4]. This descending drive to the spinal cord is diffuse and simultaneously innervates many other motor pools [14]. PICs are therefore highly sensitive to reciprocal inhibition of Ia afferents from length changes of antagonist muscles [14]. Thus, in the present study PICs attenuation may have occurred in the subjects who failed to demonstrate enhanced post-stimulation $m$. AH activation due to the postural demand required of the experimental design, without impact on PTP [5]. Therefore, five of the seven subjects who demonstrated a kinematic adaptation might well have retained the acute effects of WPS without demonstrating PICs activation. Further electrophysiological evidence is required to support this hypothesis. Whilst no significant difference was found in the EMG data, it is noteworthy that the amplitude change in $m . \mathrm{AH}$ activation immediately following WPS was $46 \%$ greater in the experimental than in the control foot of tested subjects. In comparison, only an 11\% m.AH EMG difference between feet was seen prior to WPS. This increase can be accounted for by a more than two-fold increase in $m$.AH activation observed in the two aforementioned subjects and this particular subject-specific response should not be overlooked. Taken 
together with the kinematic results, this finding suggests the potential of WPS as a modality for the prevention and treatment of common overuse foot injuries.

The present study suggests that future research with WPS in symptomatic populations is warranted. The results provided satisfactory effect sizes in the kinematic measures; furthermore they were observed at a time consistent with $m$.AH activation. The dilemma in investigating $m$.AH function under controlled conditions is the difficulty in isolating this muscle's activity. A common method for the identification of muscle-specific strength-related indices is an isometric maximal voluntary contraction (MVC). However, owing to the complexity of excluding the contribution of extrinsic and other intrinsic foot muscles to the performance measure outcomes derived from an MVC, we instead adopted a more functionally relevant approach. Therefore, whilst it would be attractive to infer that the reported kinematic changes are a direct consequence of neural plasticity, we recognise that the experimental design of the current study was not set-up sufficiently to answer this question. Finally, the present study was not designed to compare WPS with conventional electrical stimulation paradigms [9]. Indeed, the use of narrower-width pulses $(400 \mu \mathrm{s})$ has been shown equally as efficacious in augmenting acute and chronic postural responses [8]. The similarity between the aforementioned study and the present investigation however, is in the use of a highfrequency, low-intensity stimulus combined with a prolonged train to facilitate a tetanic contraction of $m . \mathrm{AH}$. Thus, the present results, combined with literature data on the use of prolonged high-frequency electrical stimulation and its relationship with the processes that facilitate PTP $[5,12]$ provide clinicians with an evidence base to pursue an interventional approach in the rehabilitation of (a)symptomatic footrelated complaints.

In summary, our findings suggest that an acute session of WPS to an intrinsic foot muscle can lead to immediate adaptation in forefoot-rearfoot coupling behaviour during walking. We propose that future research with a pathological population is warranted to investigate amenability of adaptation. Common over-use foot complaints such as plantar fasciitis are symptomatic of an inhibition of over-loaded intrinsic foot muscles; therefore, WPS to $m$.AH may be of benefit as a modality to promote muscular control during walking for loading and propulsion. These findings should be of interest to clinicians who currently adopt 
electrical stimulation therapy or those who are in search of alternative approaches to compliment

conventional methods in the rehabilitation of over-use foot injuries.

\section{References}

1. Atkinson G, Nevill AM. Statistical methods for assessing measurement error (reliability) in variables relevant to sports medicine. Sports Med 1998; 26: 217-238.

2. Caravaggi P, Pataky T, Gunther M, Savage R, Crompton R. Dynamics of longitudinal arch support in relation to walking speed: contribution of the plantar aponeurosis. J Anat 2010; 217: 254-261.

3. Chang R, Van Emmerik R, Hamill J. Quantifying rearfoot-forefoot coordination in human walking. J 345 Biomech 2008; 41:3101-3105.

4. Collins DF. Central contributions to contractions evoked by tetanic neuromuscular electrical stimulation. Exerc Sport Sci Rev 2007; 35: 102-109.

5. Dean JC, Yates LM, Collins DF. Turning off the central contribution to contractions evoked by neuromuscular electrical stimulation. Muscle Nerve 2008; 38: 978-986.

6. Del Toro DR, Park TA. Abductor hallucis false motor points: electrophysiologic mapping and cadaveric dissection. Muscle Nerve 1996; 19: 1138-1143.

7. Fiolkowski P, Brunt D, Bishop M, Woo R, Horodyski M. Intrinsic pedal musculature support of the medial longitudinal arch: an electromyography study. J Foot Ankle Surg 2003; 42: 327-333.

8. Gaillet JC, Biraud JC, Bessou M, Bessou P. Modifications of baropodograms after transcutaneous electric stimulation of the abductor hallucis muscle in humans standing erect. Clin Biomech (Bristol, Avon) 2004; 19: 1066-1069.

9. Gondin J, Brocca L, Bellinzona E, D'Antona G, Maffiuletti NA, Miotti D, Pellegrino MA, Bottinelli R. Neuromuscular electrical stimulation training induces atypical adaptations of the human skeletal muscle phenotype: a functional and proteomic analysis. J Appl Physiol 2011; 110: 433-450.

10. Hamill J, van Emmerik RE, Heiderscheit BC, Li L. A dynamical systems approach to lower extremity running injuries. Clin Biomech (Bristol, Avon) 1999; 14: 297-308.

11. Harris DJ, Atkinson G. Update--ethical standards in sport and exercise science research. Int J Sports Med 2011; 32: 819-821.

12. Hawkins RD, Kandel ER, Siegelbaum SA. Learning to modulate transmitter release: themes and variations in synaptic plasticity. Annu Rev Neurosci 1993; 16: 625-665.

13. Headlee DL, Leonard JL, Hart JM, Ingersoll CD, Hertel J. Fatigue of the plantar intrinsic foot muscles increases navicular drop. J Electromyogr Kinesiol 2008; 18: 420-425. 
15. Jam B. Evaluation and Retraining of the Intrinsic Foot Muscles for Pain Syndromes Related to Abnormal Control of Pronation.Advanced Physical Therapy Education Institute. http://www.aptei.com/articles/pdf/IntrinsicMuscles.pdf. Accessed June 26, 2012.

16. Landorf KB, Keenan AM, Herbert RD. Effectiveness of foot orthoses to treat plantar fasciitis: a randomized trial. Arch Intern Med 2006; 166: 1305-1310.

17. Leardini A, Benedetti MG, Berti L, Bettinelli D, Nativo R, Giannini S. Rear-foot, mid-foot and forefoot motion during the stance phase of gait. Gait Posture 2007; 25: 453-462.

18. Mann R, Inman VT. Phasic Activity of Intrinsic Muscles of the Foot. J Bone Joint Surg Am 1964; 46 : 469-481.

19. Mills K, Blanch P, Chapman AR, McPoil TG, Vicenzino B. Foot orthoses and gait: a systematic review and meta-analysis of literature pertaining to potential mechanisms. Br J Sports Med 2010; 44: 1035-1046.

20. Morio C, Nicol C, Barla C, Barthelemy J, Berton E. Acute and 2 days delayed effects of exhaustive stretch-shortening cycle exercise on barefoot walking and running patterns. Eur J Appl Physiol 2011, doi: 10.1007/s00421-011-2242-3.

21. Roll R, Kavounoudias A, Roll JP. Cutaneous afferents from human plantar sole contribute to body posture awareness. Neuroreport 2002; 13: 1957-1961.

22. Wong YS. Influence of the abductor hallucis muscle on the medial arch of the foot: a kinematic and anatomical cadaver study. Foot Ankle Int 2007; 28: 617-620.

23. Zehr EP, Komiyama T, Stein RB. Cutaneous reflexes during human gait: electromyographic and kinematic responses to electrical stimulation. J Neurophysiol 1997; 77: 3311-3325.

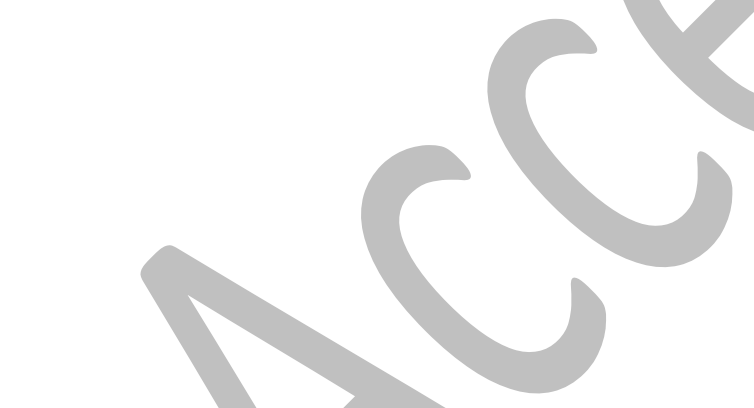

Figure 1. Illustration of the experimental procedures.

Figure 2. Subjects received 10 x 15 -s of 2-s alternating WPS $(20 \mathrm{~Hz}-100 \mathrm{~Hz}-20 \mathrm{~Hz})$ with the final $20 \mathrm{~Hz}$ stimulus in each session being 3-s in duration. EMG was analysed 2-s prior to and immediately following stimulation. In this example the subject demonstrates post-stimulation enhanced muscle activity.

Figure 3. (From Chang et al. [3]. Reprinted with permission). RF motion is plotted relative to FF for each percentile of stance. Coordination is classified as anti-phase $\left(112.5 \leq \gamma \leq 157.5 ; 292.5 \leq \gamma \leq 337.5^{\circ}\right)$, in-phase 
$404\left(22.5 \leq \gamma \leq 67.5 ; 202.5 \leq \gamma \leq 247.5^{\circ}\right)$, proximal dominance $(0 \leq \gamma \leq 22.5 ; 157.5 \leq \gamma \leq 202.5 ; 337.5 \leq \gamma \leq 360)$ and distal 405 dominance $\left(67.5 \leq \gamma \leq 112.5 ; 247.5 \leq \gamma \leq 292.5^{\circ}\right)$.

406

407 Figure 4. Frequency of observation (mean \pm SEM) of FF-RF frontal plane (left) and transverse plane

408 (right) coupling during mid-stance of the gait cycle. * denotes a significant interaction effect (foot versus 409 time [PRE versus POST]).

410

411

412

413

414

415

416

417

418

419

420 


\section{A. Motor point determination}

for $\mathrm{m}$. abductor hallucis

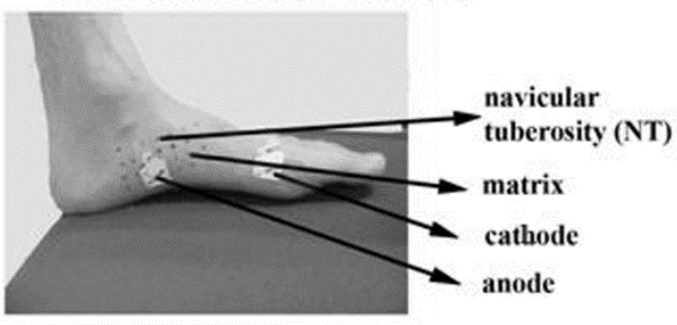

\section{B. Foot stimulation}

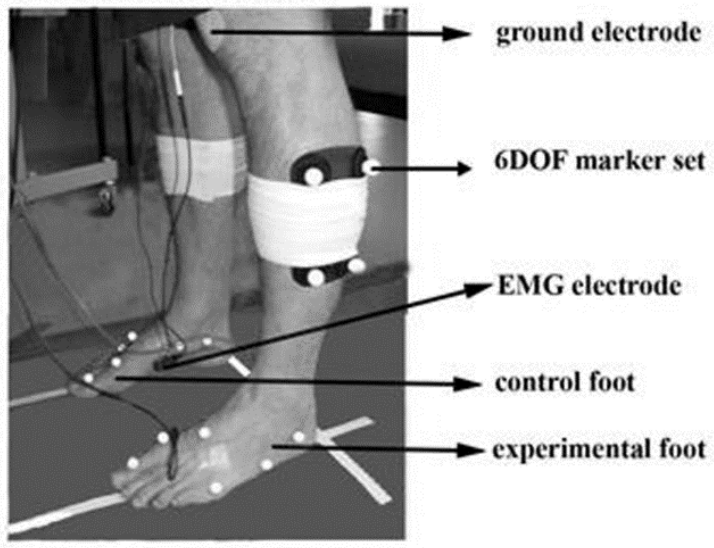

Figure 1. Illustration of the experimental procedures

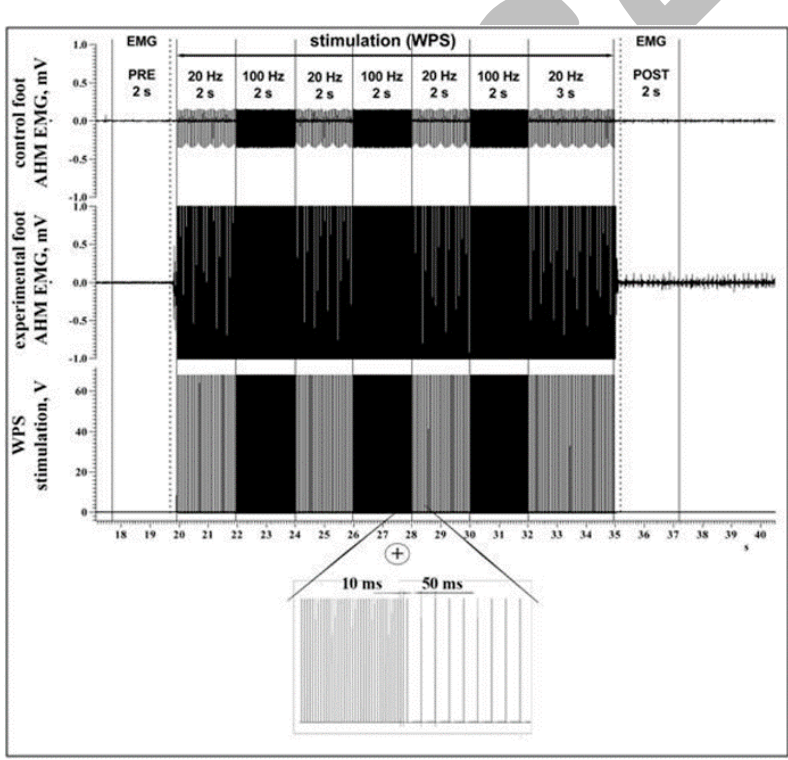

Figure 2. Subjects received $10 \times 15$-s of 2 -s alternating WPS $(20 \mathrm{~Hz}-100 \mathrm{~Hz}-20 \mathrm{~Hz})$ with the final $20 \mathrm{~Hz}$ stimulus in each session being 3-s in duration. EMG was analysed 2-s prior to and immediately following stimulation. In this example the subject demonstrates post-stimulation enhanced muscle activity. $80 \times 74 \mathrm{~mm}(300 \times 300 \mathrm{DPI})$ 


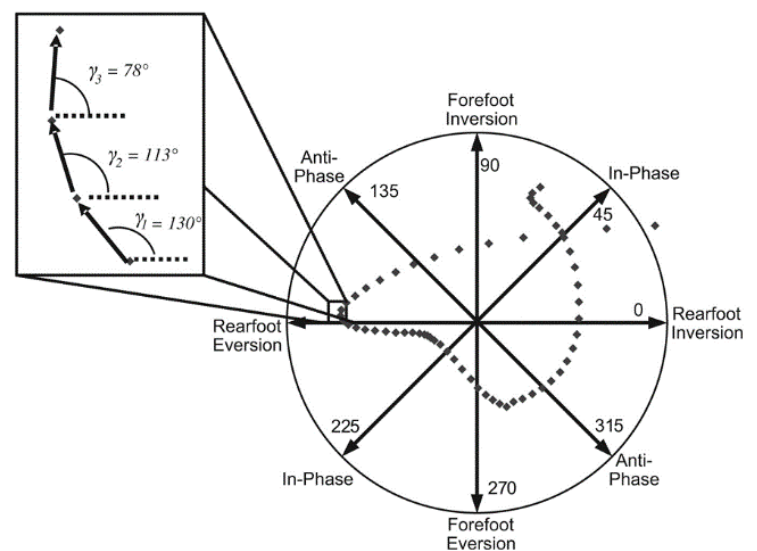

Figure 3. (From Chang et al. [3]. Reprinted with permission). RF motion is plotted relative to FF for each percentile of stance. Coordination is classified as anti-phase $\left(112.5 \leq \gamma \leq 157.5 ; 292.5 \leq \gamma \leq 337.5^{\circ}\right)$, inphase

$\left(22.5 \leq \gamma \leq 67.5 ; 202.5 \leq \gamma \leq 247.5^{\circ}\right)$, proximal dominance $(0 \leq \gamma \leq 22.5 ; 157.5 \leq \gamma \leq 202.5 ; 337.5 \leq \gamma \leq 360)$ and

distal dominance $\left(67.5 \leq y \leq 112.5 ; 247.5 \leq y \leq 292.5^{\circ}\right)$. $80 \times 57 \mathrm{~mm}(300 \times 300 \mathrm{DPI})$
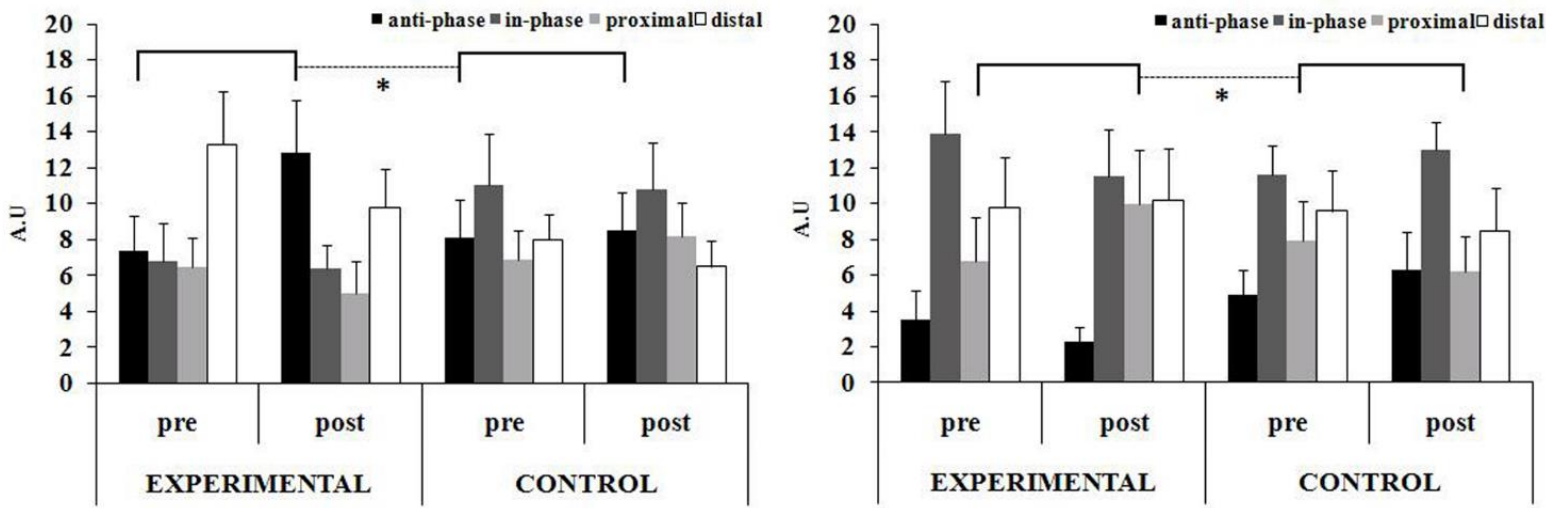

Figure 4. Frequency of observation (mean \pm SEM) of FF-RF frontal plane (left) and transverse plane (right)

coupling during mid-stance of the gait cycle. * denotes a significant interaction effect (foot versus time [PRE

versus POST]).

$170 \times 57 \mathrm{~mm}(300 \times 300 \mathrm{DPI})$ 